\title{
Mid-infrared interferometry of the massive young stellar object NGC 3603 - IRS 9A
}

\author{
S. Vehoff ${ }^{1,2}$, C. A. Hummel ${ }^{3}$, J. D. Monnier ${ }^{4}$, P. Tuthill ${ }^{5}$, D. E. A. Nürnberger ${ }^{2}$, \\ R. Siebenmorgen ${ }^{3}$, O. Chesneau ${ }^{6}$, and W. J. Duschl ${ }^{7,8}$
}

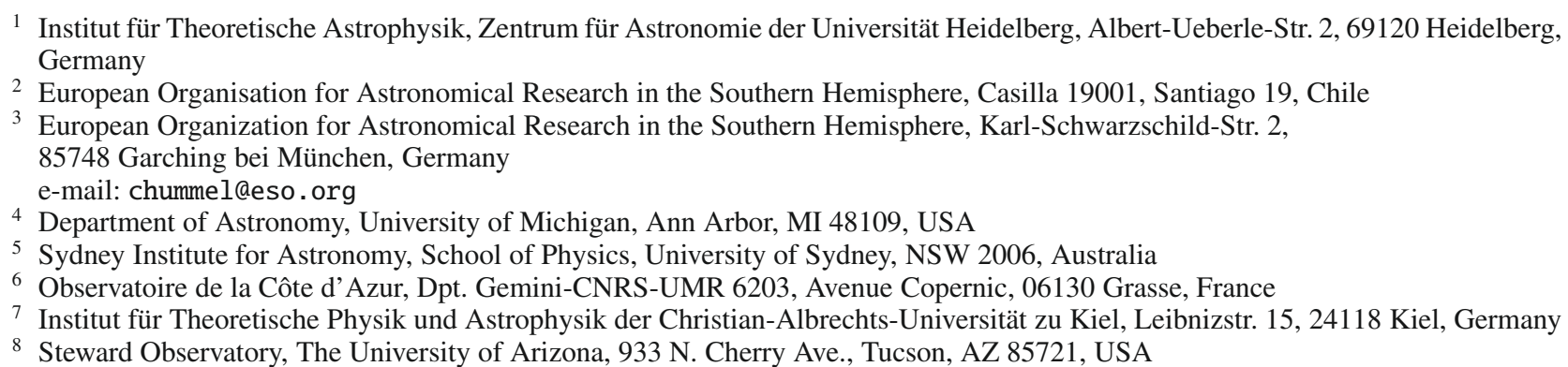

Received 26 October 2009 / Accepted 7 July 2010

\section{ABSTRACT}

\begin{abstract}
Context. Very few massive young stellar objects (MYSO) have been studied in the infrared at high angular resolution due to their rarity and large associated extinction. We present observations and models for one of these MYSO candidates, NGC 3603 IRS 9A. Aims. Our goal is to investigate with infrared interferometry the structure of IRS 9A on scales as small as 200 AU, exploiting the fact that a cluster of $\mathrm{O}$ and $\mathrm{B}$ stars has blown away much of the obscuring foreground dust and gas.

Methods. Observations in the $N$-band were carried out with the MIDI beam combiner attached to the VLTI, providing spatial information on scales of about 25-95 milli-arcsec (mas). Additional interferometric observations which probe the structure of IRS 9A on larger scales were performed with an aperture mask installed in the T-ReCS instrument of Gemini South. The spectral energy distribution (SED) is constrained by the MIDI $N$-band spectrum and by data from the Spitzer Space Telescope. Our efforts to model the structure and SED of IRS 9A range from simple geometrical models of the brightness distribution to one- and two-dimensional radiative transfer computations.

Results. The target is resolved by T-ReCS, with an equivalent (elliptical) Gaussian width of 330 mas by 280 mas (2300 AU by 2000 AU). Despite this fact, a warm compact unresolved component was detected by MIDI which is possibly associated with the inner regions of a flattened dust distribution. Based on our interferometric data, no sign of multiplicity was found on scales between about $200 \mathrm{AU}$ and $700 \mathrm{AU}$ projected separation. A geometric model consisting of a warm $(1000 \mathrm{~K})$ ring (400 AU diameter) and a cool $(140 \mathrm{~K})$ large envelope provides a good fit to the data. No single model fitting all visibility and photometric data could be found, with disk models performing better than spherical models.

Conclusions. While the data are clearly inconsistent with a spherical dust distribution they are insufficient to prove the existence of a disk but rather hint at a more complex dust distribution.
\end{abstract}

Key words. techniques: interferometric - circumstellar matter - stars: early-type - stars: formation - stars: pre-main sequence - stars: individual: NGC 3603 IRS 9A

\section{Introduction}

The formation and evolution of massive stars is so rapid that by the time the star has reached the zero-age main sequence, it is still enshrouded in large amounts of gas and dust of the molecular cloud from which it was born. Thus, high extinction is usually present at this stage and prevents the optical identification and classification of the star, as well as a more detailed study of the inner regions of the circumstellar dust. Furthermore, highmass stars are rare objects and hence generally much more distant than low-mass star forming regions, requiring high angular resolution for study. For a detailed account of the theoretical and

* Based in part on observations collected at the European Southern Observatory, Chile (Prop. No. 074.C-0062) and the Gemini South Observatory, Chile. observational issues connected to the formation of (high-mass) stars we refer to the review articles by McKee \& Ostriker (2007) and Zinnecker \& Yorke (2007).

In the case of formation and early evolution of low-mass (T Tauri) stars and intermediate-mass (Herbig Ae/Be) stars, the longer timescales involved allow us to study the mass accretion process, the chemical evolution of the dust in the regions close to the star, and the subsequent dispersion of the circumstellar material in great detail. A key ingredient of current models is the formation of a disk which plays an important role in removing angular momentum from the infalling material. Thus, one would expect a similar scenario for the formation of high-mass stars, even more so since the problem of their extreme radiation pressure acting upon the spherical dust envelopes would limit their final mass (e.g., Wolfire \& Cassinelli 1987; Krumholz et al. 2009). 
But it appears that one cannot simply scale the low-mass star formation models to higher masses (Zinnecker \& Yorke 2007), since, amongst other reasons, most, if not all, high-mass stars are born in cluster environments (e.g., de Wit et al. 2005) where accretion might occur in a competitive manner (Bonnell et al. 1997, 2001; Clark et al. 2008).

The target of our study, [FPA77] NGC 3603 IRS 9A, has been identified as one of the most luminous mid-infrared sources located within the giant HII region NGC 3603 (Nürnberger 2003). This star forming region is dominated by one of the densest and most massive clusters of $\mathrm{O}$ and $\mathrm{B}$ type main sequence stars in the Galaxy (Moffat et al. 1994). The stellar winds and ionising radiation of these stars have blown away most of the gas and dust of the molecular cloud core around IRS 9A (Nürnberger 2008), which lies at a projected distance of only $2.5 \mathrm{pc}$ from the central cluster. In spite of a distance towards NGC 3603 of about $(7 \pm 1) \mathrm{kpc}$, the visual foreground extinction towards the cluster is only 4-5 mag (Sher 1965; Moffat 1983; Melnick et al. 1989). Using infrared colour-magnitude diagrams and pre-main sequence evolutionary tracks, Nürnberger (2003) was able to deduce a mass of $\approx 40 M_{\odot}$ for IRS 9 A. Its luminosity was estimated to about $2.3 \times 10^{5} L_{\odot}$ and its age to $\approx 10^{4} \mathrm{yr}$. The spectral index $\alpha_{2.2-10 \mu \mathrm{m}}=1.37$ is similar to those of (low-mass) IR class I objects, indicating that IRS 9A is still embedded in significant amounts of gravitationally bound material.

These properties all argue for IRS 9A's classification as a high-mass young stellar object which, due to its advantageous location, can be studied more easily. Observations at very highangular resolution in the mid-infrared hold the potential to yield valuable constraints for the theoretical understanding of highmass star formation, including multiplicity, but only very few other MYSOs have been studied via (mid-) infrared interferometry so far. Among them are W33A (de Wit et al. 2007), M8EIR (Linz et al. 2009), and MWC 297 (Acke et al. 2008). We will compare the properties of IRS 9A to these objects later on in this work.

The remaining sections of this paper are organised as follows. In Sect. 2 we present the main results of our observations, shortly discussing the data reduction processes and immediate implications of the individual results. In Sect. 3 we model the circumstellar structure of IRS 9A using simple geometrical models and dedicated radiative transfer models. Finally, we discuss the results of these efforts in Sect. 4 and present our conclusions in Sect. 5.

\section{Observations and data reduction}

\section{1. $M I D I$}

Even though the MID-infrared Interferometric instrument (MIDI) on the VLTI is primarily an interferometric instrument, photometry and imaging are by-products of each observation. Thus, a total flux spectrum can be obtained as well as images which are taken during the target acquisition procedure. Due to the use of an adaptive optics system (MACAO) at the Coudé foci of each UT of the VLT, these images can be analysed for medium scale structures of the target. In the following, we will describe each of these three data products.

\subsubsection{Interferometry}

The interferometric observations using MIDI (Leinert et al. 2003b,a) were carried out in February and March of 2005. The most important details of these observations are summarised in
Table 1. Log of observations of IRS 9A with MIDI, all performed in 2005.

\begin{tabular}{ccccccc}
\hline \hline Date & $\begin{array}{c}\text { Time } \\
\text { (UT) }\end{array}$ & Target & $\begin{array}{c}B_{\mathrm{p}} \\
{[\mathrm{m}]}\end{array}$ & $\begin{array}{c}\lambda / B_{\mathrm{p}} \\
{[\mathrm{mas}]}\end{array}$ & $\begin{array}{c}\text { BLPA } \\
{\left[{ }^{\circ}\right]}\end{array}$ & AM \\
\hline Feb. 27 & 08h 01m & HD 107446 & 38.0 & 43.4 & 60.6 & 1.3 \\
& 08h 35m & IRS 9A & 31.8 & 52.0 & 79.6 & 1.5 \\
& 09h 16m & HD 107446 & 33.6 & 49.1 & 74.0 & 1.4 \\
Feb. 28 & 06h 24m & HD 107446 & 41.8 & 39.5 & 44.3 & 1.2 \\
(a) & 06h 48m & IRS 9A & 38.0 & 43.5 & 60.6 & 1.3 \\
& 07h 09m & HD 107446 & 40.2 & 41.0 & 52.2 & 1.2 \\
(b) & 07h 43m & IRS 9A & 34.9 & 47.3 & 70.6 & 1.4 \\
& 08h 04m & HD 107446 & 37.6 & 43.8 & 61.8 & 1.3 \\
(c) & 08h 33m & IRS 9A & 31.6 & 52.2 & 80.0 & 1.5 \\
& 08h 54m & HD 107446 & 34.8 & 47.5 & 70.7 & 1.4 \\
(d) & 09h 21m & IRS 9A & 28.0 & 58.9 & 89.9 & 1.7 \\
& 09h 48m & HD 107446 & 31.0 & 53.2 & 80.8 & 1.5 \\
Mar. 3 3 & 04h 21m & HD 107446 & 56.2 & 29.5 & 81.4 & 1.3 \\
& 05h 27m & HD 107446 & 59.4 & 27.8 & 95.8 & 1.3 \\
& 07h 10m & IRS 9A & 62.5 & 26.5 & 131.9 & 1.3 \\
& 07h 49m & HD 107446 & 62.5 & 26.5 & 126.0 & 1.3 \\
& 08h 54m & IRS 9A & 61.9 & 26.7 & 155.5 & 1.6 \\
& 09h 16m & HD 107446 & 62.2 & 26.5 & 145.2 & 1.5 \\
& 09h 33m & HD 107446 & 62.0 & 26.6 & 149.1 & 1.5 \\
& 09h 51m & HD 107446 & 61.9 & 26.7 & 153.3 & 1.6 \\
\hline
\end{tabular}

Notes. The spatial resolution $\lambda / B_{\mathrm{p}}$ is calculated for $8 \mu \mathrm{m}$. BLPA $=$ baseline position angle projected on sky (east of north), $\mathrm{AM}=$ airmass. The letters (a)-(d) refer to Fig. 2.

Table 1 . The resulting $(u, v)$-coverage is shown in Fig. 1 together with the coverage resulting from the T-ReCS observations. The synthesized interferometric beam size is about 28 mas by 16 mas, PA of 47 degrees. The observations of NGC 3603 IRS 9A were interleaved with measurements of HD 107446, our calibrator, for which we adopted a diameter of 4.7 mas based on the $(V-K)$ color index (Mozurkewich et al. 2003). A star with this diameter is nearly unresolved on a $40 \mathrm{~m}$ baseline and at a wavelength of $8 \mu \mathrm{m}\left(V^{2}=0.97\right)$. HD 107446's angular distance to IRS 9A is about $8^{\circ}$; it has a spectral type of K3.5III and an IRAS flux of $32.4 \mathrm{Jy}$ in the $12 \mu \mathrm{m}$ filter. MIDI was used with the high-sensitivity beam combiner configuration (just two interferometric outputs) and the prism for spectral dispersion, providing a spectral resolution of $R \approx 35$.

The data were reduced using the MIA+EWS (version 1.5) software package ${ }^{1}$ which is described in detail in Ratzka (2005). In order to calculate the visibility, the correlated flux is normalised with the total flux. The latter is obtained directly after the interferometric measurements, using the individual telescopes and chopping between the target position and a sky position (for IRS 9A: position angle $=117^{\circ}$, throw $=17^{\prime \prime}$ ). The calibration of the target visibilities was achieved by division with the calibrator visibilities, interpolated for each epoch of the target observations. We estimated the calibration uncertainty from the scatter of several measurements of the calibrator on Feb. 28 to range from about $10 \%$ at $13 \mu \mathrm{m}$ to about $20 \%$ at $8 \mu \mathrm{m}$. The resulting calibrated visibilities of IRS 9A are shown in Fig. 2 (UT2-UT3 baseline) and in Fig. 3 (UT3-UT4 baseline). The single visibility spectrum of Feb. 27 is not shown as it is redundant, and the second observation of Mar. 3 was taken at high airmass and did not yield data of good quality. The data are made available at $\mathrm{OLBIN}^{2}$.

\footnotetext{
1 Available at:

http://www.strw. leidenuniv.nl/ nevec/MIDI/index.html

2 http://olbin.jpl.nasa.gov/data/
} 


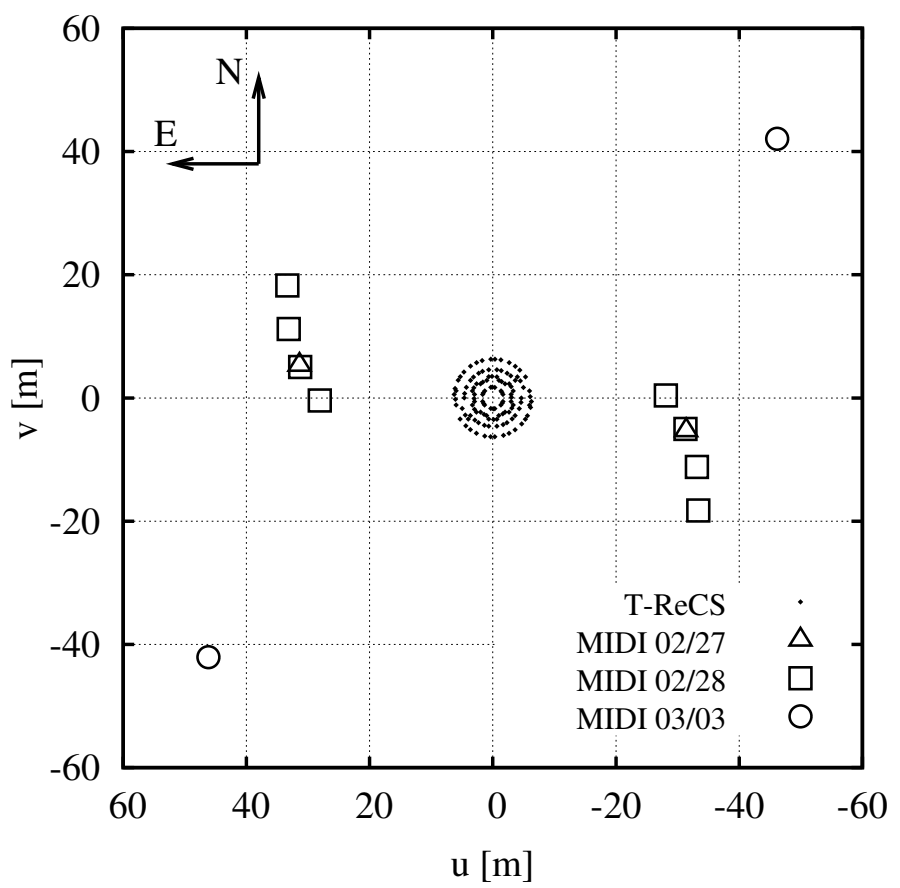

Fig. 1. $(u, v)$-plane coverage of the interferometric observations with MIDI at the VLTI and the aperture masking observations with T-ReCS at Gemini South.

(a)

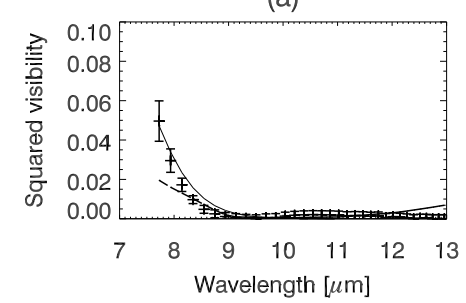

(b)

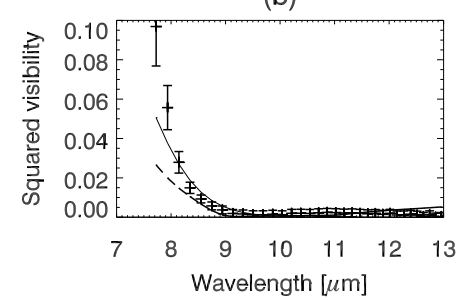

Fig. 2. Squared visibilities measured with MIDI on the UT2-UT3 baseline on Feb. 28, 2005, with letters (a)-(d) corresponding to the four observations listed in Table 1. The solid lines are best fits of the geometrical model described in Sect. 3.1, and the dashed lines are from the physical model described in Sect. 3.3.

The visibilities are very similar for all our baselines. They drop to virtually zero for wavelengths larger than about $9 \mu \mathrm{m}$ where IRS 9A appears fully resolved by MIDI. Yet we detect a compact component (roughly 60 mas in diameter) which emerges below this wavelength, causing a steep rise of the visibilities.

\subsubsection{Spectro-photometry}

Since HD 107446 is not a spectro-photometric calibrator, we adopted for its spectrum the template of HD 163588 (K2III) from the list of spectro-photometric standards by

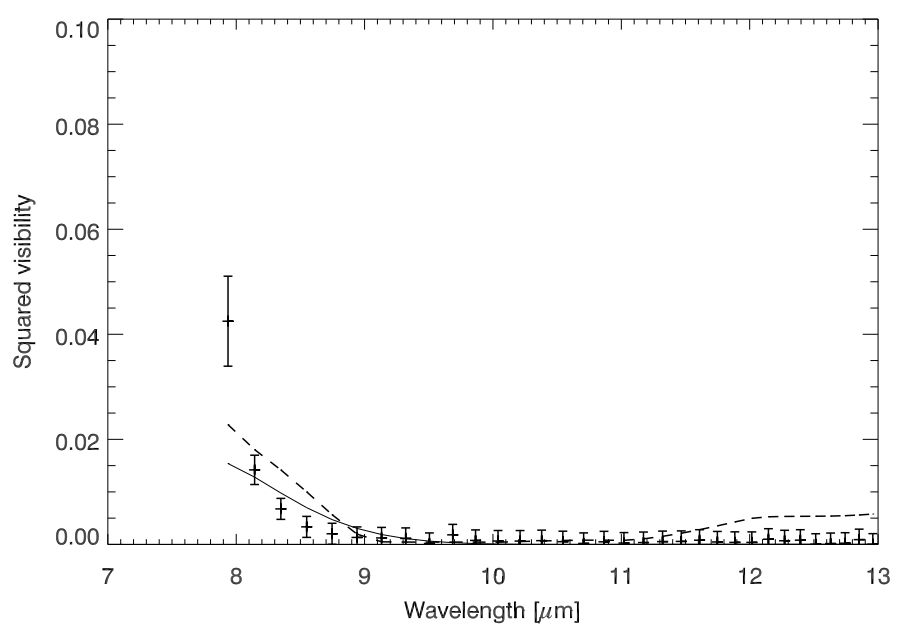

Fig. 3. Squared visibilities measured with MIDI on the UT3-UT4 baseline (first observation on Mar. 3, 2005). The solid line is a best fit obtained from the geometrical model described in Sect. 3.1, and the dashed line is from the physical model described in Sect. 3.3.

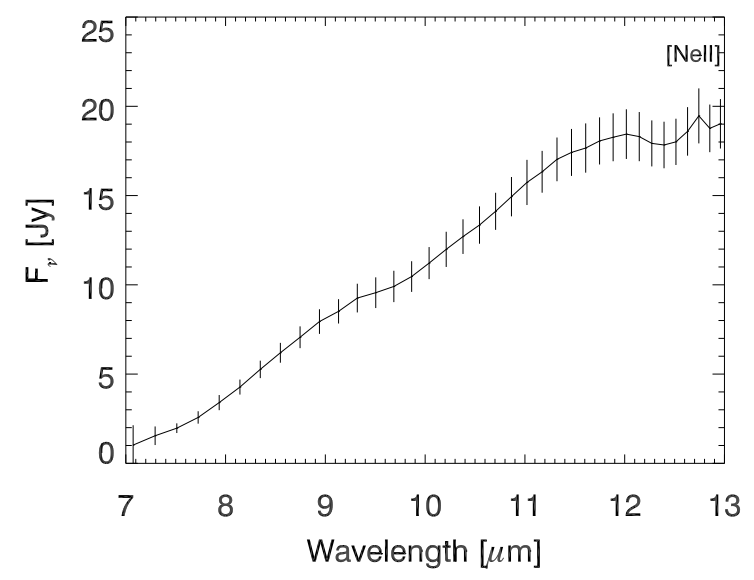

Fig. 4. Average spectrum of IRS 9A, as obtained from the MIDI data taken on Feb. 28, 2005. The small spike at $12.8 \mu \mathrm{m}$ coincides with the [Ne II] line.

Cohen et al. (1999), and used the ratio of the IRAS $12 \mu \mathrm{m}$ fluxes to determine the relative scale. The resulting flux-calibrated spectrum of IRS 9A as measured with MIDI is shown in Fig. 4. We averaged only spectra from Feb. 28, 2005 as they represent the largest homogeneous data set. The error bars are based on the standard deviation of the spectra.

There are several interesting aspects concerning this spectrum. First, the flux of IRS 9A rises steeply with increasing wavelength. Second, there is no sign of a silicate feature at about $9.7 \mu \mathrm{m}$, neither in absorption nor in emission. This is rather uncommon for dust-enshrouded objects like YSOs. Third, despite the low spectral resolution of MIDI, there is a hint of the [Ne II] emission line at $12.8 \mu \mathrm{m}$, prominently seen by Spitzer (see Sect. 2.3).

\subsubsection{Imaging}

The chopped acquisition images of IRS 9A were taken with the $\mathrm{N} 8.7$ filter $\left(\lambda_{\mathrm{c}}=8.64 \mu \mathrm{m}, \lambda_{F W H M}=1.54 \mu \mathrm{m}\right)$ and have a spatial 


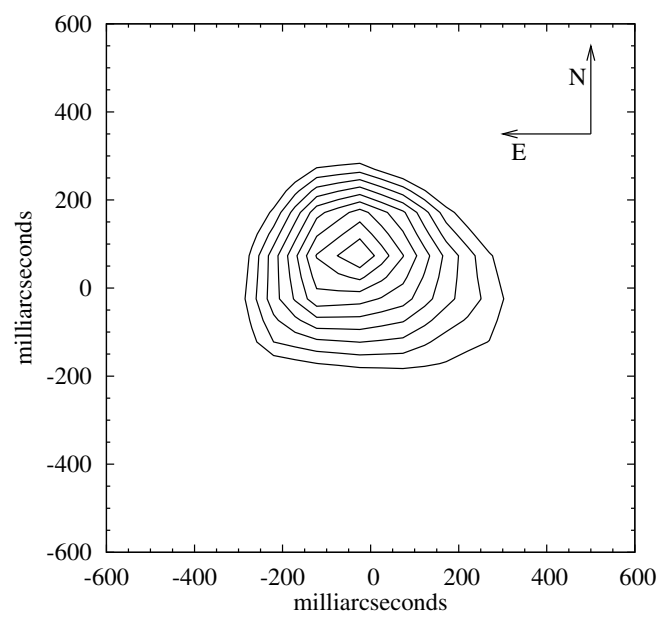

Fig. 5. Deconvolved MIDI acquisition image of IRS 9A in the N8.7 filter after 25 iterations. The contour lines are equally spaced for the fourth root of the intensity and lie at 3.7, 6.3, 10, 15, 22, 32, 44, 59 and 77 percent of the maximum intensity.

resolution of about $0.3^{\prime \prime}$. Using the calibrator HD 107446 as a PSF reference, the images can be deconvolved to further enhance the resolution, using the procedure described by Chesneau et al. (2005). We used the Lucy-Richardson algorithm (Lucy 1974) for this purpose, taken from the IDL Astronomy Library ${ }^{3}$. However, we detected a residual jitter in our images caused by a delay in the acquisition by the adaptive optics loop each time the chop position changed. Simply re-centering the frames did not lead to useful results due to distorted profiles while MACAO was closing the loop. Therefore, we only stacked the chopped frames which were well centered (about half of the frames).

We stopped the algorithm after 25 iterations, a compromise between image convergence and fitting noise. A representative example of our deconvolved acquisition images is shown in Fig. 5. We performed two-dimensional Gaussian fits to the deconvolved images in order to determine the FWHM sizes and the orientation of the major axis. The results are summarised in Table 2 which lists the mean single night values, and also the mean value of all our observations, using the number of observations per night as the associated weight $\left(N_{\text {obs }}\right)$. We did not use the images of the beam B of MIDI (UT3 on Feb. 27 and Feb. 28, UT4 on Mar. 3) due to their slightly inferior image quality and the associated systematically larger FWHM values. Overall, IRS 9A has a Gaussian FWHM of about 389 mas by 354 mas, and a position angle of the major axis of about 75 degrees (east of north). Despite the different values of the parallactic angle of the observations, i.e. the direction to north on the detector, ranging from 130 to 173 degrees, the derived PA of the fitted ellipse was unchanged. Representing the overall shape of the source, the fitting of the PA was apparently dominated by the $\mathrm{SW}$ ridge in the source structure.

\section{2. $T-\operatorname{ReCS}$}

We also observed NGC 3603 IRS 9A with the T-ReCS (ThermalRegion Camera Spectrograph, de Buizer \& Fisher 2005) instrument at the Gemini South Observatory on January 2, 2005. A 7-hole non-redundant aperture mask was used to reduce the weight of the short spacings realised within the $8 \mathrm{~m}$ aperture of the telescope, and therefore to yield high quality images at the

3 http://idlastro.gsfc.nasa.gov/

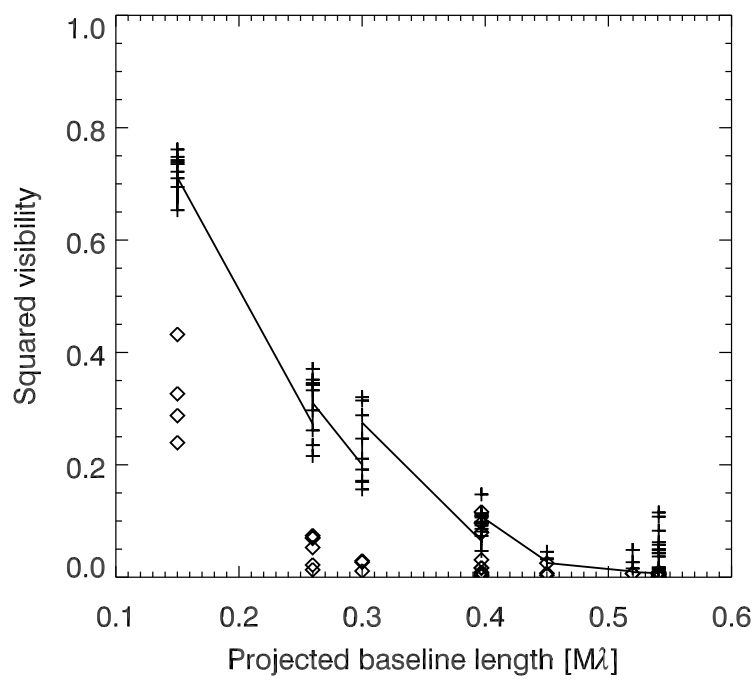

Fig. 6. Squared visibility amplitudes from T-ReCS plotted versus projected baseline length. Error bars have been omitted for clarity; they are shown in Fig. 7. The vertical scatter of the visibility amplitudes arises from non-axisymmetric structure, which causes some azimuthal dependence of the amplitudes. The solid line is a best fit obtained from the geometrical model described in Sect. 3.1, and the diamonds correspond to the physical model described in Sect. 3.3.

Table 2. Results of the Gaussian fits to the deconvolved MIDI acquisition images.

\begin{tabular}{cccccc}
\hline \hline Date & Beam & $N_{\text {obs }}$ & $\begin{array}{c}\text { Major } \\
{[\mathrm{mas}]}\end{array}$ & $\begin{array}{c}\text { Minor } \\
{[\mathrm{mas}]}\end{array}$ & $\begin{array}{c}\text { PA } \\
{\left[{ }^{\circ}\right]}\end{array}$ \\
\hline Feb. 27, 2005 & UT2 & 1 & 402 & 339 & 82 \\
Feb. 28, 2005 & UT2 & 4 & 392 & 366 & 71 \\
Mar. 03, 2005 & UT3 & 2 & 375 & 339 & 81 \\
Mean values & & 7 & 389 & 354 & 75 \\
\hline
\end{tabular}

diffraction limit (for a description of the aperture masking technique see, e.g., Monnier 1999; Tuthill et al. 2000). The data were taken with the $\operatorname{Si}-5$ filter $\left(\lambda_{\mathrm{c}} \pm \Delta \lambda=11.66 \mu \mathrm{m} \pm 0.57 \mu \mathrm{m}\right)$. The resulting $(u, v)$-coverage is shown in Fig. 1; the synthesized beam is almost circular with a diameter of 264 mas.

The interferograms, which result from the superposition of all interference patterns between any combination of apertures in the mask, are Fourier transformed to measure the visibility amplitudes and phases. This is done for both target and calibrator, and the calibration procedure is similar to the one used in long baseline interferometry. The resulting squared visibility amplitudes are shown in Figs. 6 and 7. The data are made available at $\mathrm{OLBIN}^{4}$.

In order to derive an image from the set of amplitudes and phases, we employed the VLBMEM imaging software (Sivia 1987), which uses a Maximum Entropy algorithm. The resulting image is shown in Fig. 8. It agrees well with the overall appearance of our MIDI acquisition images, again showing the emission of IRS 9A to be quite extended and slightly asymmetric.

\subsection{Spitzer}

In Fig. 9, we show the SED of IRS 9A measured by Lebouteiller et al. (2007, 2008) with the Spitzer Space Telescope in

\footnotetext{
${ }^{4}$ http://olbin.jpl.nasa.gov/data/
} 


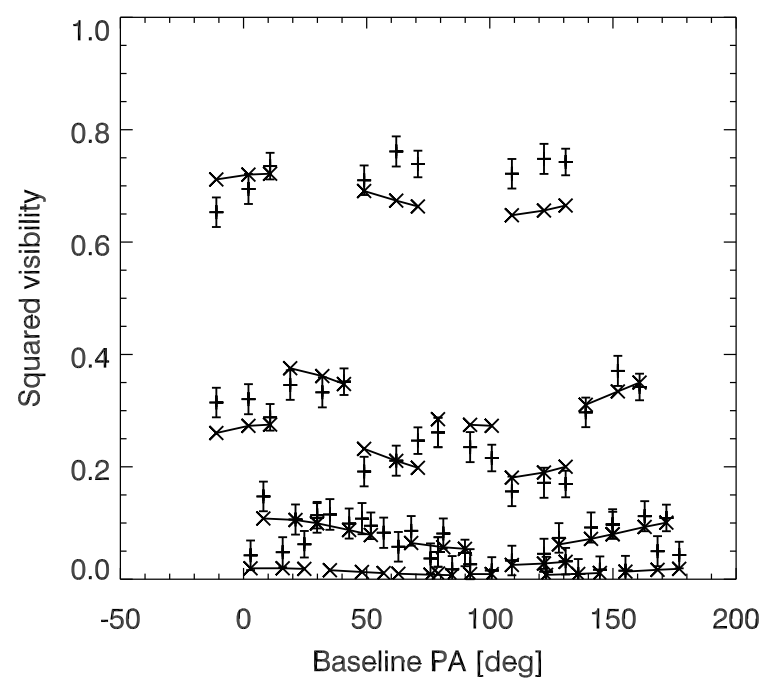

Fig. 7. Squared visibility amplitudes from T-ReCS (+-symbols) plotted versus baseline position angle $(0-180)$. The vertical offsets of the visibility amplitudes is due to the different lengths of baselines with the same position angle. The solid lines (with $\times$-symbols) are the best fits obtained from the geometrical model described in Sect. 3.1.

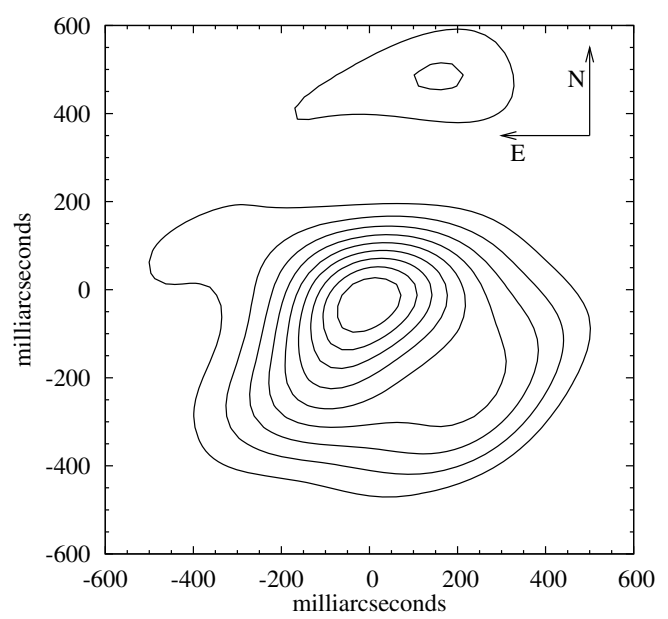

Fig. 8. T-ReCS image of IRS 9A at $11.7 \mu \mathrm{m}$. North is up, and east is to the left. Reconstruction from the aperture masking data using the Maximum Entropy Method (MEM). Contour levels are the same as in Fig. 5.

comparison with the MIDI spectrum. Since Spitzer's spatial resolution is about a factor of 10 worse compared to the (MACAOassisted) VLT, the IRS spectrograph aboard Spitzer collects considerably more flux, about twice that of MIDI in the middle of the $\mathrm{N}$ band. There is no hint of the [S IV] line in our MIDI data, although its line flux is more than two times higher than the one from the [Ne II] line (see Lebouteiller et al. 2008) which we did barely detect with MIDI (see Fig. 4). It is possible that the region emitting the [S IV] line is more extended than the MIDI field of view. We will use the Spitzer SED as an additional constraint for our models in Sect. 3.

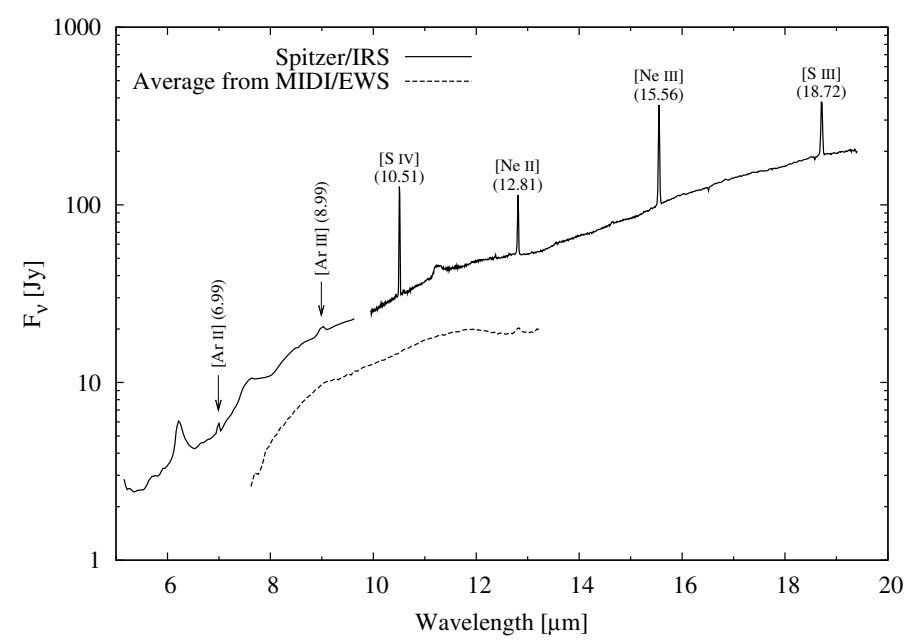

Fig. 9. The spectrum of IRS 9A from Spitzer (solid lines) and MIDI (dotted line). The numerous forbidden emission lines are labelled together with their central wavelengths. There are also several PAH features visible, with the most prominent ones lying at 6.2, 7.6 and $11.2 \mu \mathrm{m}$. The small gap is due to saturation of Spitzer's SL module for wavelengths larger than $9.7 \mu \mathrm{m}$. The data from the LH module (18.7$37.2 \mu \mathrm{m}$ ) suffered from the same effect and are not shown (see also Lebouteiller et al. 2008).

\section{Model fitting and analysis}

In this section, we first try to model the circumstellar structure of NGC 3603 IRS 9A in the context of current star formation scenarios by using such elements as rings, disks, and envelopes. We then describe our efforts of testing physical models which require radiative transfer computations to produce images of the structure for comparison with the available visibility and photometric data. However, given that the environment of young and very luminous stars is complex, our models can only represent a first step on the way of developing an adequate physical model of IRS 9A. Also the paramerters of these models may not be well constrained given their large number.

\subsection{Simple geometrical models}

It is useful to identify structural components of the source and derive the parameters of such simple geometrical models like sizes and orientations. Beginning with the visibilities measured with T-ReCS in a single filter (hence no wavelength dependence of the source structure need to be considered), we first fit circular Gaussian and uniform disk models with diameters of 311 mas $(F W H M)$ and 495 mas, respectively. The reduced $\chi^{2}$ (henceforth denoted with $\chi_{\mathrm{r}}^{2}$ ) of the Gaussian model can be further reduced to 2.3 by allowing an ellipsoid, with a major axis of 329 mas, PA of $100^{\circ}$ east of north, and a minor axis of 282 mas (axial ratio 0.86, compare with values from Table 2). This component would be completely resolved by MIDI even on the shortest baselines available, and therefore a second component of small angular scale needs to be added to the geometrical model in order to explain the MIDI detection. After trying unsuccessfully to fit the data with an additional uniform disk, we adopt a ringshaped component instead because its Fourier transform exhibits a larger secondary maximum (beyond the null) than a uniform disk. The ring, which could be associated with the inner hot regions of a disk with a central hole, is initially assigned a blackbody temperature of $1500 \mathrm{~K}$ (equal to the typical dust sublimation temperature) and a fractional width of $1 / 4$ of its radius. We 


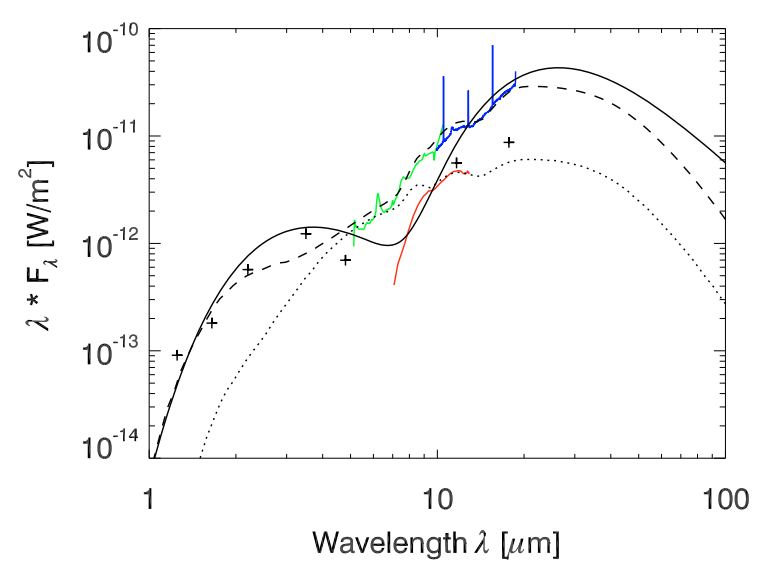

Fig. 10. The SED of the geometrical model (solid line) with the observations from Spitzer (green and blue solid lines), MIDI (red solid line), and NIR/MIR photometry (plus symbols) published by Nürnberger (2003). The two components of the model have the same flux at 8 microns. The dashed and dotted lines correspond to the physical model described in Sect. 3.3, with aperture radii of 3" (Spitzer) and 0.3" (MIDI), respectively.

Table 3. The parameters of the geometrical model.

\begin{tabular}{llr}
\hline \hline Parameter & Unit & Value \\
\hline Ring major axis & {$[\mathrm{mas}]$} & 57 \\
Major axis PA & $\left.{ }^{\circ}\right]$ & $105^{a}$ \\
Ratio minor/major axis & & 0.32 \\
Fractional ring width & & 0.31 \\
Ring effective temperature & {$[\mathrm{K}]$} & 1000 \\
Envelope major axis $F W H M$ & {$[\mathrm{mas}]$} & 350 \\
Major axis PA & $\left.{ }^{\circ}\right]$ & $105^{a}$ \\
Ratio minor/major axis & & 0.86 \\
Envelope eff. temperature & {$[\mathrm{K}]$} & 140 \\
\hline
\end{tabular}

Notes. ${ }^{(a)}$ Forced to be the same value.

assume here, before a more realistic physical model is applied later on, that the two components are co-located and do not obscure each other. In order to have the flux of the Gaussian component decrease towards the blue end of the N-band so that the correlated flux of the ring begins to dominate here the visibility measured by MIDI (see Fig. 10), we assign initially a low blackbody temperature of $250 \mathrm{~K}$ to the extended Gaussian component based on a spectral decomposition by Nürnberger (2003). This model ("geometrical model") indeed results in a good fit $\left(\chi_{\mathrm{r}}^{2}=1.6\right)$ of all visibility data (see Figs. 2 and 3 , as well as Figs. 6 and 7), with the best-fit parameter values given in Table 3. The inclination of the polar axis of the ring against the line of sight is 71 degrees. The fit to the SED is shown in Fig. 10.

There is no clear evidence for a companion of IRS 9A, and we can use the geometrical model to estimate at what level we can exclude a companion. We adopt the companion to be a ring like the primary so that the binary model asymptotically approaches the geometrical model for small separations when the individual component fluxes are adjusted (given a magnitude difference) to yield a constant total flux. For the limiting $\chi_{\mathrm{r}}^{2}$, we adopted 1.7 which results in recognizable deviations from the data. Almost all models below this limit have a magnitude difference of 3 or larger, and therefore we can exclude the possibility that a companion of more than about $0.5 \mathrm{Jy}$ exists between 30 mas (the resolution limit) and 500 mas (our search area) away from the primary. Due to the fact that uncorrelated flux dominates at the longer wavelengths where none of the models could

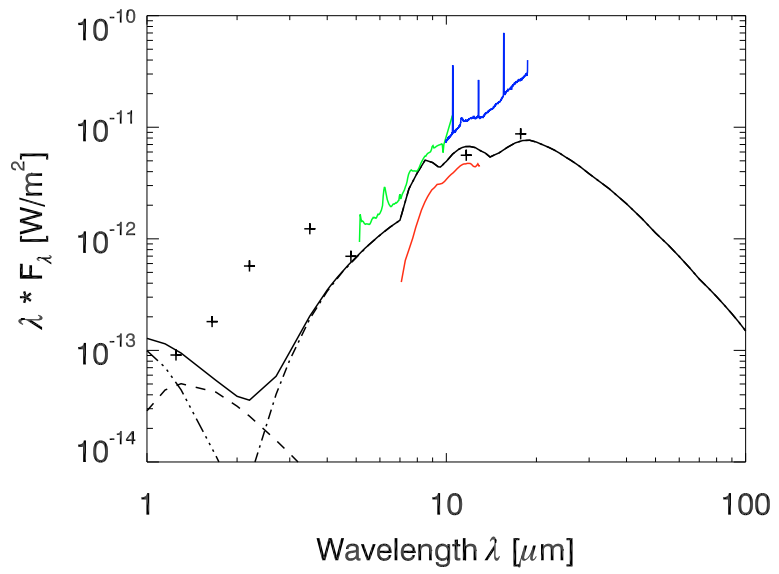

Fig. 11. Comparison of the SED of our DUSTY model with the observations from Spitzer, MIDI, and the NIR/MIR photometry (plus symbols) from Fig. 10. The dashed line corresponds to the attenuated input radiation, the dash-dotted line to the dust radiation, the dash-double-dotted line to the scattered emission.

therefore produce any significant signatures, we cannot place more stringent limits.

\subsection{Spherical dust shell models}

We now interpret the geometrical model we have studied as spherical dust shells with the innermost one corresponding to the ring component and with the star at their center. For the calculation of the radiative transfer we use the DUSTY code (Ivezić \& Elitzur 1997, 1999; Ivezić et al. 1999) which adopts spherical symmetry. It produces detailed spectra and images at specified wavelengths, and the latter can be used to calculate the visibilities of the models that correspond to the observational setup of T-ReCS or MIDI. These types of models have been used quite successfully by de Wit et al. (2007) and de Wit et al. (2009) for their MIDI observations of W33A and resolved $24.5 \mu \mathrm{m}$ emission of MYSOs, respectively.

For the central star we adopted an effective temperature of $22000 \mathrm{~K}$, and for the shells a density, $\rho$, proportional to $r^{-2}$, where $r$ is radius of the shell. For the dust composition we used standard astronomical silicates. The most important free parameters to adjust were the temperature, $T_{\mathrm{s}}$, of the inner edge of the shells, and its opacity, $\tau$. In general, a lower $T_{\mathrm{s}}$ increased the size of the shells and thus lowered the visibility amplitudes, while a larger opacity had a similar effect yet introduced a strong silicate absorption feature in the spectrum. Using the T-ReCS data and the SED as constraints, we settled on a model with $T_{\mathrm{s}}=500 \mathrm{~K}$ and $\tau_{10 \mu \mathrm{m}}=1.5$, corresponding to $A_{V}=29 \mathrm{mag}$ (Rieke \& Lebofsky 1985). The fit to the T-ReCS visibilities was indistinguishable from the (circular) Gaussian model, while the fit to the SED is shown in Fig. 11. DUSTY returns the radius of the innermost shell, $r_{\text {in }}$ (where $T=T_{\mathrm{s}}$ ), which scales proportional with the square root of the luminosity, $L^{1 / 2}$. Adopting a luminosity of IRS 9A of $230000 L_{\odot}$, we derive $r_{\mathrm{s}}=170$ mas. The (dust) mass of the shell can be derived from the opacity $\tau$, the absorption coefficient $\kappa$, and the ratio of the outer edge of the shell to $r_{\text {in }}, Y$, as follows,

$$
\tau=\int_{r_{\text {in }}}^{r_{\text {out }}} \kappa \rho \mathrm{d} s=\rho_{0} \kappa \frac{r_{\text {in }}}{r_{\text {out }}}\left(r_{\text {out }}-r_{\text {in }}\right),
$$


$M=\int_{r_{\text {in }}}^{r_{\text {out }}} \rho 4 \pi r^{2} \mathrm{~d} r=4 \pi \frac{\tau}{\kappa} r_{\text {in }}^{2} Y$,

giving a dust mass of about $1 M_{\odot}$.

We thus find that it is possible to devise a physical model for the T-ReCS data set, but when we apply that model to the observations from MIDI, the agreement is very poor because the emission is completely resolved spatially. This also applies viceversa, that is we are able to find models with DUSTY which can reproduce the visibilities measured by MIDI (albeit showing larger deviations), but these models are in turn a very poor fit to the data from T-ReCS. In order to find suitable models that are able to account for all our observational data, we conducted a grid search varying all parameters except for $T_{\mathrm{s}}, Y$ and the temperature of the central star. However, none of the resulting models could accomplish this task.

It thus seems obvious that spherical dust distributions are not compatible with the circumstellar structure of IRS 9A. Therefore, in the next section, we examine the effects of nonspherical dust distributions on the visibilities and the SED.

\subsection{Disk models}

Within the framework of models for YSOs, the most obvious approach for a non-spherical density distribution is given by the flattened structure of a circumstellar disk embedded in an envelope. If seen under low (near face-on) or intermediate inclinations of the disk axis to the line of sight, a disk opens a more or less direct optical path to the central object and the hot inner disk regions (the hot ring in our geometrical model), while at the same time maintaining an extended dust distribution (the cool Gaussian component of the geometrical model). Models involving disks have already been used widely for interpreting SEDs and interferometric observations of $\mathrm{T}$ Tauri and Herbig Ae/Be stars. For example, the visibilities predicted by models computed for Herbig Ae/Be stars by Leinert et al. (2004) look similar to our MIDI visibilities. In this section we report on our first attempt to fit such models to our data.

We adopt the disk-envelope model of Whitney et al. (2003b) which has been used by Robitaille et al. (2006) to compute a large grid of model SED, made available through a web server ${ }^{5}$. Following Linz et al. (2009), we select only those models fitting the Spitzer-SED of IRS9A and compute their images. To compute the model visibilities, we apodized the images at scales larger than the PSF of an $8 \mathrm{~m}$ telescope in the case of MIDI, while due to the comparitively small subapertures in TReCS, this apodization was not necessary. Thus we find that the model 3012790 inclined at $85^{\circ}$ to the line of sight (nearly edge on) and with the disk roughly oriented East-West provides the best fit of the ten selected models. The parameters of this model are listed in Table 4. The disk-envelope model 3012790 includes a significant accretion rate, for which however no independent constraint exists so far. The fits of this model to the visibilities are shown in Figs. 2, 3, and 6, while the SED is shown in Fig. 10.

\section{Discussion}

Considering the MIDI observations, the most important feature to reproduce by any model is the steep rise of the visibility towards 8 microns, indicating the emergence of warm inner dusty regions. The disk-envelope model is able to reproduce this feature about as well as the geometrical model, but the envelope

5 http://caravan.astro.wisc.edu/protostars/
Table 4. The parameters of the Robitaille disk-envelope model 3012790.

\begin{tabular}{llr}
\hline \hline Parameter & Unit & Value \\
\hline Stellar mass & {$\left[M_{\odot}\right]$} & 25 \\
Stellar radius & {$\left[R_{\odot}\right]$} & 6.5 \\
Effective temperature & {$[\mathrm{K}]$} & 38000 \\
Luminosity & {$\left[\mathrm{L}_{\odot}\right]$} & 92000 \\
Inner disk/envelope radius & {$[\mathrm{AU}]$} & 25 \\
Outer disk radius & {$[\mathrm{AU}]$} & 94 \\
Outer envelope radius & {$[\mathrm{AU}]$} & 100000 \\
Disk dust mass & {$\left[M_{\odot}\right]$} & 0.005 \\
Envelope dust mass & {$\left[M_{\odot}\right]$} & 0.9 \\
Inclination & {$\left[{ }^{\circ}\right]$} & 85 \\
Disk flaring power, $\beta$ & & 1.2 \\
Disk scale height & {$[\mathrm{AU}]$} & 9 \\
Cavity cone angle & {$\left[{ }^{\circ}\right]$} & 29 \\
\hline
\end{tabular}

is too large considering the low predicted visibilities for the TReCS observations.

The T-ReCS image of IRS 9A (Fig. 8) resembles the synthetic images of class I sources computed by Whitney et al. (2003a, their Fig. 11a) and indicates the presence of a bipolar cavity facing the observer and pointing towards the South to South-West. Ionizing stellar radiation escaping through the cavity has created an HII region as indicated by the detection of the [Ne II] line by Spitzer (see Fig. 9). This morphology of the circumstellar dust (and gas) distribution is consistent with the interpretation of NACO images of NGC 3603 IRS 9A by Nürnberger (2008) which show an envelope with a major-to-minor axis ratio of about $2: 1$ at a position angle of $82^{\circ}$. (The NACO observations also confirm the reality of the faint feature in our T-ReCS image about 0.6 arcseconds to the north of IRS 9A.) Due to the limited dynamic range of our images, we cannot confirm the ablation of dust and gas towards the south-east, i.e. away from the NGC 3603 cluster center, as seen in the NACO images.

A lower mass estimate of $0.1 M_{\odot}$ for the hot dust and gas bound to IRS9A was derived by Nürnberger (2003) based on the assumption that the MIR emission is optically thin. For the envelope mass, an estimate of $1 M_{\odot}$ is derived by the same author based on an estimate of the intrinsic extinction of $A_{\mathrm{V}}=10-15$, much lower than our estimate based on DUSTY. However, Nürnberger (2003) remarked that a radio flux measurement of NGC 3603 IRS 9A yielded unexpectedly high values, which would argue in favour of a higher envelope mass estimate. A final answer cannot be given until a sub-mm flux measurement can be obtained.

\section{Conclusions}

We gave a detailed account of our efforts to model the observed spatial and spectral data of the MYSO candidate NGC 3603 IRS 9A. As a first result, we find that the observed visibilities are not compatible with a binary nature of IRS 9A. However, given the limits in sensitivity $(\approx 0.5 \mathrm{Jy}$; the correlated flux on longer baselines would have been too small to detect) and in angular resolution (down to 30 mas) of our interferometric data, we cannot exclude the presence of a companion with either significantly lower mid-infrared flux (less than 10\%) and/or an extremely tight binary with a separation of less than about $100 \mathrm{AU}$.

We have shown that a spherical dust distribution is inconsistent with the data and that therefore a flattened distribution is more likely. A geometrical model consisting of a warm ring and a cool extended component can reproduce all observations fairly 
well, but requires the ad hoc assumption of a direct line of sight into the inner regions of the warm dust. The ring has a temperature $500 \mathrm{~K}$ less than the dust sublimation temperature $(1500 \mathrm{~K})$, probably a consequence of the fact that it represents not only the very inner edge of a disk, but rather the inner regions of it. Physical disk-envelope models provide a self-consistent connection between the hot inner and the cooler outer regions and allow the former to be seen more directly. However, no single model was able to fit all observations simultaneously. Since it could be expected from the early evolutionary stage of class I sources compared to the well developed disks of the class II sources (adopting the classification scheme more strictly developed for lower mass objects), that the dust structures are more complex, e.g. clumpy or showing disk distortions, our data are not sufficient to prove the existence of a disk.

Comparing NGC 3603 IRS 9A to other MYSOs for which MIDI interferometry was published, e.g. W33A (de Wit et al. 2007), M8E-IR (Linz et al. 2009), and MWC 297 (Acke et al. 2008), we find that all of them exhibit rather different properties. These depend mostly on the amount of circumstellar material present, as reflected in the estimated visual extinctions which range from $A_{\mathrm{V}}=22$ to $A_{\mathrm{V}}=100$. The source with the highest extinction, W33A, displays a deep silicate absorption feature. The measured visibilities were similar to the ones we measured between 8 and $9 \mu \mathrm{m}$ on the same baseline, translating into a Gaussian FWHM of 120 AU for W33A (distance of $3.8 \mathrm{kpc}$ ). A significant difference here to NGC 3603 IRS 9A is that the latter is completely resolved by MIDI at longer wavelengths due to the envelope detected with T-ReCS. Thus, W33A seems a lot more compact, and was therefore successfully modelled with a single DUSTY envelope. The model required a rather low effective stellar temperature for a $10 M_{\odot}$ star (only $10000 \mathrm{~K}$ ), possibly indicative of a star swollen due to accretion. The same effect was observed in M8E-IR, which was modelled with an envelope and disk surrounding a rather cool $(5000 \mathrm{~K})$ star with a mass of $13.5 M_{\odot}$. M8E-IR has a much weaker silicate absorption feature, and appears to be rather compact (45-100 AU). The existence of a disk in the envelope could not be ascertainted. Yet again, the difference to NGC 3603 IRS 9A lies in the fact that the visibility of M8E-IR is only weakly dependent on wavelength, indicating that a flattened structure is not necessary to explain them. Finally, the observations of MWC 297 could not be modeled with a physical disk model at all. This star is a young $10 M_{\odot}$ Be star with a rather low extinction. Only indirect arguments favoured the disk geometry for the circumstellar material.

Acknowledgements. We are grateful to Vianney Lebouteiller for kindly providing the Spitzer spectrum of IRS 9A. We also thank Mario van den Ancker for sharing his insight during several discussions of this work. S.V. acknowledges support from DFG via SFB 439 and from the University of Kiel, Germany. J.D.M. acknowledges support from NASA NNG05GI80G, NSF-AST0352728. Some observations contained herein were obtained at the Gemini Observatory, which is operated by the Association of Universities for Research in Astronomy, Inc., under a cooperative agreement with the NSF on behalf of the Gemini partnership: the National Science Foundation (United States), the Science and Technology Facilities Council (United Kingdom), the National Research Council (Canada), CONICYT (Chile), the Australian
Research Council (Australia), Ministério da Ciência e Tecnologia (Brazil) and Ministerio de Ciencia, Tecnología e Innovación Productiva (Argentina). This research has made use of the SIMBAD database, operated at CDS, Strasbourg, France.

\section{References}

Acke, B., Verhoelst, T., van den Ancker, M. E., et al. 2008, A\&A, 485, 209 Bonnell, I. A., Bate, M. R., Clarke, C. J., \& Pringle, J. E. 1997, MNRAS, 285, 201

Bonnell, I. A., Bate, M. R., Clarke, C. J., \& Pringle, J. E. 2001, MNRAS, 323, 785

Chesneau, O., Verhoelst, T., Lopez, B., et al. 2005, A\&A, 435, 563

Chiang, E. I., \& Goldreich, P. 1997, ApJ, 490, 368

Clark, P. C., Klessen, R. S., Bonnell, I. A., \& Smith, R. J. 2008, in Massive Star Formation: Observations Confront Theory, ed. H. Beuther, H. Linz, \& T. Henning, ASP Conf. Ser., 387, 208

Cohen, M., Walker, R. G., Carter, B., et al. 1999, AJ, 117, 1864

de Buizer, J., \& Fisher, R. 2005, in High Resolution Infrared Spectroscopy in Astronomy, ed. H. U. Käufl, R. Siebenmorgen, \& A. Moorwood, 84 de Wit, W. J., Testi, L., Palla, F., \& Zinnecker, H. 2005, A\&A, 437, 247 de Wit, W. J., Hoare, M. G., Oudmaijer, R. D., \& Mottram, J. C. 2007, ApJ, 671, L169

de Wit, W. J., Hoare, M. G., Fujiyoshi, T., et al. 2009, A\&A, 494, 157

Dullemond, C. P., Dominik, C., \& Natta, A. 2001, ApJ, 560, 957

Ivezić, Ž., \& Elitzur, M. 1997, MNRAS, 287, 799

Ivezić, Ž., \& Elitzur, M. 1999, MNRAS, 303, 864

Ivezić, Ž., Nenkova, M., \& Elitzur, M. 1999, User Manual for DUSTY, Internal report, University of Kentucky, accessible at http://www.pa.uky.edu/ moshe/dusty

Krumholz, M. R., Klein, R. I., McKee, C. F., Offner, S. S. R., \& Cunningham, A. J. 2009, Science, 323, 754

Lebouteiller, V., Brandl, B., Bernard-Salas, J., Devost, D., \& Houck, J. R. 2007, ApJ, 665, 390

Lebouteiller, V., Bernard-Salas, J., Brandl, B., et al. 2008, ApJ, 680, 398

Leinert, C., Graser, U., Richichi, A., et al. 2003a, The Messenger, 112, 13 Leinert, C., Graser, U., Waters, L. B. F. M., et al. 2003b, in Interferometry for Optical Astronomy II, ed. W. A. Traub, SPIE Conf. Ser., 4838, 893

Leinert, C., van Boekel, R., Waters, L. B. F. M., et al. 2004, A\&A, 423, 537

Linz, H., Henning, T., Feldt, M., et al. 2009, A\&A, 505, 655

Lucy, L. B. 1974, AJ, 79, 745

McKee, C. F., \& Ostriker, E. C. 2007, ARA\&A, 45, 565

Melnick, J., Tapia, M., \& Terlevich, R. 1989, A\&A, 213, 89

Moffat, A. F. J. 1983, A\&A, 124, 273

Moffat, A. F. J., Drissen, L., \& Shara, M. M. 1994, ApJ, 436, 183

Monnier, J. D. 1999, Ph.D. Thesis, University of California, Berkeley

Mozurkewich, D., Armstrong, J. T., Hindsley, R. B., et al. 2003, AJ, 126, 2502

Nürnberger, D. E. A. 2003, A\&A, 404, 255

Nürnberger, D. E. A. 2008, J. Phys. Conf. Ser., 131, 012025

Ratzka, T. 2005, Ph.D. Thesis, University of Heidelberg, Germany

Rieke, G. H., \& Lebofsky, M. J. 1985, ApJ, 288, 618

Robitaille, T. P., Whitney, B. A., Indebetouw, R., Wood, K., \& Denzmore, P. 2006, ApJS, 167, 256

Schegerer, A. A., Wolf, S., Ratzka, T., \& Leinert, C. 2008, A\&A, 478, 779

Schegerer, A. A., Wolf, S., Hummel, C. A., Quanz, S. P., \& Richichi, A. 2009, submitted

Sher, D. 1965, MNRAS, 129, 237

Sivia, D. S. 1987, Ph.D. Thesis, Cambridge University

Tuthill, P. G., Monnier, J. D., Danchi, W. C., Wishnow, E. H., \& Haniff, C. A. 2000, PASP, 112, 555

Whitney, B. A., Wood, K., Bjorkman, J. E., \& Cohen, M. 2003a, ApJ, 598, 1079 Whitney, B. A., Wood, K., Bjorkman, J. E., \& Wolff, M. J. 2003b, ApJ, 591, 1049

Wolf, S. 2003, Comp. Phys. Commun., 150, 99

Wolf, S., Henning, T., \& Stecklum, B. 1999, A\&A, 349, 839

Wolfire, M. G., \& Cassinelli, J. P. 1987, ApJ, 319, 850

Zinnecker, H., \& Yorke, H. W. 2007, ARA\&A, 45, 481 